\title{
Repetition blindness in priming in perceptual identification: Competitive effects of a word intervening between prime and target
}

\author{
Jennifer S. Burt ${ }^{1}$ • Jessica Jolley ${ }^{1}$
}

Published online: 23 June 2017

(C) Psychonomic Society, Inc. 2017

\begin{abstract}
University students named a 72-ms masked target word that was preceded by two 120 -ms consecutively presented words, a prime word followed by a distractor. In Experiment 1, all words were in lowercase letters, whereas in Experiment 2, the target word was changed to uppercase letters. In both experiments there was an accuracy and latency cost (repetition blindness: RB) when the prime was the same word as the target, with the cost much less severe in Experiment 2 than in Experiment 1. A low-frequency distractor impaired target identification compared with a high-frequency distractor. Distractor frequency interacted with target frequency such that high-frequency targets preceded by low-frequency distractors had the lowest accuracy. The results are consistent with a frequency-dependent competition for access to working memory among briefly displayed words. However, there was no clear evidence that effects of target repetition on interword competition play a role in RB. The effects of a letter case change for the target are consistent with a contribution of token distinctiveness to word-order recovery in the intervening-word priming task.
\end{abstract}

Keywords Attention $\cdot$ Lexical processing $\cdot$ Repetition priming

Repetition blindness (RB) refers to the deficit in reporting a repeated item in a rapid sequence of items displayed visually in a fixed location (rapid serial visual presentation; RSVP). With word stimuli, the focus of interest here, there have been

Jennifer S. Burt

j.burt@psy.uq.edu.au

1 School of Psychology, University of Queensland, Brisbane, QLD 4072, Australia many demonstrations of RB, typically with an item duration of around $100 \mathrm{~ms}$ (Chialant \& Caramazza, 1997; Kanwisher, 1987; Kanwisher \& Potter, 1990). In the original studies, the word sequence was a sentence for complete report. In simplified paradigms, the number of to-be-reported words is small, and some or all of the intervening items might be random letter or symbol strings. For example Bavelier, Prasada and Segui (1994) had one intervening word between C1 and C2, or one intervening word and one symbol string. In all arrangements, a robust deficit in $\mathrm{C} 2$ accuracy is observed when $\mathrm{C} 1$ and $\mathrm{C} 2$ are identical.

Theories of RB and related effects focus on limitations on individual event registration under time pressure, refractoriness in the lexical system, competition for awareness amongst stream items, or memory reconstructive processes. Kanwisher and colleagues (Kanwisher, 1987; Kanwisher \& Potter, 1989) devised a theory about event registration that distinguished type representations, preexisting memory representations that support item identification, and tokens, episodic instantiations of a particular occurrence of a type. Registering an event requires binding a type to a token. On their model there is a limit on the rate of type-token binding, with types not available for a new token binding immediately after a binding has been made. The second account relies on refractoriness in the type node, that is, a reduced responsiveness for a brief period after the type node has been activated (Bond \& Andrews, 2008; Huber, 2008; Luo \& Caramazza, 1996). Refractoriness was excluded by Kanwisher (1987) as a viable account of RB because participants reporting just the last stream item (C2) showed facilitation of report when $\mathrm{C} 1$ was identical to $\mathrm{C} 2$, suggesting preactivation of the type representation. However, this result was not replicated, with RB reported by others in this task (Whittlesea, Dorken, \& Podrouzek, 1995).

Competition is the core principle of a third theoretical approach, which is the primary focus of the present research. 
Morris, Still, and Caldwell-Harris (2009) supposed that in RSVP, given the absence of sustained activation of each item, there is competition between a current item and an immediately preceding or following item for access to awareness. It is supposed that access can be achieved for only one item at a time. $\mathrm{RB}$ is attributed to a reduced competitiveness resulting from the effect of repetition on an item's activation. Specifically, their model is inspired by the reduction in the neural response to a repeated stimulus, termed repetition suppression (Grill-Spector, Henson, \& Martin, 2006). Morris et al. (2009) note that although a recently presented item invokes a sharper activation response, with a resulting increase in the signal-to-noise ratio that facilitates identification, there is a reduction in the total activation (signal plus noise). Under time pressure, this reduction in total activation puts the item at a disadvantage in competition with other stimuli. By contrast, an unrepeated stimulus, which evokes a noisier response but a larger summed activation than a repeated stimulus, is better able to compete with items for access to awareness in an RSVP task. Competitiveness is also affected by repetition in the long term, with well-learned (high-frequency) words having sparser activation than low-frequency words. As outlined later, we varied word frequency to vary word competitiveness.

Memory reconstructive accounts differ from the above accounts in that they locate the RB effect at least partly at the end of the stream during preparation of response rather than attributing it to processes occurring during the RSVP stream. They suppose that participants use a number of sources of information to infer event identity and order (Whittlesea \& Masson, 2005).

Important background to the present research is the inconsistency in results (RB vs. a repetition benefit) when participants respond to a repeated word. In lexical priming tasks, participants typically see a single prime word that requires no response, and then name a target or classify it as a word versus nonword (lexical decision task; LDT). When the target remains in view until a response is made, repetition benefits are universal over a large range of prime-target stimulus onset asynchronies (SOAs: Burt, Kipps, \& Matthews, 2014; Forster $\&$ Davis, 1984). However, when the target in a priming task is displayed briefly (say, less than $100 \mathrm{~ms}$ ), repetition benefits at short SOAs may change to repetition costs (RB), typically when the prime duration exceeds about $400 \mathrm{~ms}$ (Burt et al., 2014; Huber, 2008). These conflicting effects of repetition and the role of prime duration are not well understood.

RB studies in RSVP, unlike priming studies, normally have a minimum of one item between $\mathrm{C} 1$ and $\mathrm{C} 2$, presumably to allow them to be separated perceptually. As in the RSVP, the present experiments had an intervening item between the prime $(\mathrm{C} 1)$ and target $(\mathrm{C} 2)$. As in lexical priming paradigms, the only response required of participants was naming the target word. The duration of words was chosen to be within the range used in the RSVP. We expected the present studies to confirm our expectation that RB is the typical result when only the target is named, provided that the target duration is brief (Burt et al., 2014).

In previous work (Leggett et al., unpublished) we presented primes and following distractors for $120 \mathrm{~ms}$ each, and found that when the distractor was a word, an identical prime produced a substantial repetition cost. This result is reminiscent of studies in the RSVP by Whittlesea and Masson (2005), who found that RB was substantially greater when filler items were different words versus repetitions of a single word (white) or symbol strings. In their experiments, participants had to decide whether there was a repetition in the RSVP stream or decide how many times a probe word had occurred. The authors argued that keeping track of targets to detect repetitions was cognitively demanding, and that word fillers increased the memory and decision load. In the studies of Leggett et al., there was no requirement to find repeats, suggesting that there may be additional reasons for the difficulty posed by word distractors. Specifically, interitem competition (Morris et al., 2009) may contribute to the substantial RB found for word distractors but not random-letter distractors.

The present experiments investigated the role of intervening distractor words by varying the frequency of the distractor and the target (together with its prime). Frequency has large effects on visual word identification (Monsell, 1991). Critically, with respect to RB, the Morris et al. model supposes that increasing word frequency, like repetition, reduces an item's competitiveness with an adjacent distractor word. The substantial number of learning exposures to a high-frequency word produce a more finely tuned activation profile in a word detector (type node), compared with the noisier response, but larger summed activation, evoked by a low-frequency word. The aims of the present studies were, first, to ascertain whether there is evidence of frequency-dependent competition in the identification of a brief target, and, second, to seek evidence that such competition plays a role in RB.

In terms of competitive effects of the distractor word, predictions are based on the likelihood that the distractor will win in a competition for awareness with the target and cause a failure to report the target. A straightforward expectation is that the intervening distractor is more likely to win the competition against a target whose frequency is low than a target whose frequency is high. Furthermore, by extension, a lowfrequency target should better resist distractor competition than a high-frequency target. If the theory is correct with respect to RB, competition by the distractor should modulate $\mathrm{RB}$. Whether RB also interacts with target word frequency on the Morris et al. (2009) model depends upon on the overall magnitude of $\mathrm{RB}$, so precise predictions about an $\mathrm{RB} \times$ Frequency interaction cannot easily be made. More detailed predictions follow.

The present two experiments were conducted concurrently with random assignment to experiment. They differed only in 
the letter case for the target word. The rationale for varying letter case was that although $\mathrm{RB}$ is thought to be preserved over later case differences for C1 and C2 (Kanwisher, 1987), pilot work in our task indicated that a letter-case change enhances target distinctiveness and thus potentially reduces RB. Therefore the two experiments provided an opportunity to test competition effects under different RB magnitudes.

\section{Experiment 1}

In Experiment 1, the three successive words, prime, distractor and target, were displayed in lowercase letters. The duration of the target was set at $72 \mathrm{~ms}$, compared with $120 \mathrm{~ms}$ for the prime and distractor. In line with a report by Whittlesea and Masson (2005), we found in pilot testing that accuracy was at ceiling when the target duration was $120 \mathrm{~ms}$. The participants' task was to name the final word (the target).

The target and distractor frequency were factorially varied in a within-participants design over two levels (high vs. low), with the prime frequency matching the target frequency. Primes were either identical to the target or unrelated.

$\mathrm{RB}$ was predicted on the basis of our previous work and evidence that $\mathrm{RB}$ can be observed when participants report only the last word (Burt et al., 2014; Huber, 2008; Whittlesea et al., 1995). Kanwisher (1987) argued that RB would not be expected in this task because tokenization is not required, but this assumption may not hold in general. With respect to priming paradigms, there is uncertainty with respect to prime duration, in view of findings that RB was observed only when the prime duration exceeded about $400 \mathrm{~ms}$ (Huber, 2008). In Huber's studies, in contrast to RSVP studies of RB, there was no intervening item between a word and its repetition. The additional word may increase the difficulty of registering separate events and also increases the similarity of the priming task to RSVP. Thus, we judged $\mathrm{RB}$ to be more likely than repetition-based facilitation.

As noted above, if Morris et al. (2009) are correct about interitem competition, then low-frequency distractors should produce lower target accuracy than high-frequency distractors. Furthermore, if interitem competition is a key factor in RB, and assuming no floor effects on accuracy, then a Distractor Frequency $\times$ Repetition interaction can be expected, with larger RB for low- than for high-frequency distractors. Specifically, repeated targets can be expected to show a reduction in competitiveness that is exacerbated when the preceding distractor word is a more competitive (i.e., lowfrequency) word. A three-way interaction would be consistent with the Morris et al. model if it took the form of a larger RB with a low-frequency distractor, but only when target frequency was high. A high-frequency target is more vulnerable to competition than a low-frequency target, and when repeated, a high-frequency target might suffer a larger RB as a result of more often losing the competition against a low-frequency distractor.

In terms of target-word frequency effects, a clear prediction from the lexical processing literature is superior performance for high- than for low-frequency words. By contrast, the Morris et al. (2009) model predicts that low-frequency target words are more effective in interitem competition by virtue of their large, noisy activation footprint. Therefore, on this model, contrary to the straightforward prediction from the lexical processing literature, superior performance for high-frequency targets may be abolished by competition with the preceding distractor. A Distractor Frequency $\times$ Target Frequency interaction is possible: A reverse frequency effect (superior performance for low frequency targets) is most likely to be observed when distractor competition is strong, that is, when the distractor frequency is low.

There is little evidence on the interactive effects of target frequency and RB. A previous study found RB to be additive with word frequency (Bavelier et al., 1994), and, as noted, the only theory that predicts an interactive effect of target frequency and repetition acknowledges that whether additive or interactive effects of frequency are found depends upon the magnitude of RB (Morris et al., 2009).

\section{Method}

Participants Twenty-four undergraduate psychology students (19 female, mean age 19.08 years) participated for course credit.

Materials and design One hundred and twenty-eight target words were chosen from the British National Corpus (BNC; Kilgarriff, 1995) to fill high- and low-frequency sets ( $N=64$ each), with half of the targets in each set four letters and half five letters in length. The high-frequency targets had a frequency of at least 90 per million (mean 371), and the lowfrequency targets had a frequency range of one to 12 per million (mean 5.7). High- and low-frequency targets were matched on the number of orthographic neighbors $(N=6.1)$. An unrelated length-matched prime was taken for each target from the BNC. The unrelated primes covered a similar frequency range as the targets and approximately matched them on mean frequency in the BNC (391 per million for highfrequency primes and 5.1 per million for low-frequency primes). Finally, 64 high- and 64 low-frequency words were chosen from the BNC as intervening distractor words. As before, each set was divided equally into four- and five-letter words, and their frequency distribution approximately matched that of targets. The mean frequency was 470 per million (high-frequency distractors) and 5.3 per million (low-frequency distractors).

An additional 54 four- and five-letter words of medium frequency (mean 30 per million) were selected from the 
$\mathrm{BNC}$ to make up 12 filler trials and six practice trials. In all of these trials, the prime and target were unrelated.

The high- and low-frequency item sets were divided into four subsets of 16 matched on length composition and cycled through four conditions over four counterbalanced lists. The four lists operationalized a $2 \times 2 \times 2$ design for Distractor Frequency $\times$ Target Frequency $\times$ Prime Type, with frequency as a between-item variable. A target was seen with an identity prime in two lists and with the same unrelated word for the unrelated prime condition in the remaining two lists. In one of the lists for each prime condition, a high-frequency word was assigned as the distractor, and in the other list, a low-frequency word was assigned as the distractor. Within each level of distractor frequency, the same distractor word was used for a target in each prime condition (identity and unrelated). The filler trials were added to each list and the trial sequence was randomized, and the six practice trials were placed at the beginning of each list.

Procedure Participants were tested individually on a PC and monitor, with the experiment controlled by an E-Prime program (Schneider, Eschman, \& Zuccolutto, 2002). They wore a headset microphone that was connected to the computer via a voice-operated relay. Words were presented in lowercase Courier New font, in white lettering on a black screen. On each trial, a ready signal (+++) was displayed in the center of the screen for $350 \mathrm{~ms}$, and then the prime, distractor, and target were presented in succession in the same location. The prime and distractor durations were $120 \mathrm{~ms}$, and the target duration was $72 \mathrm{~ms}$. The target was followed by a pattern mask (\%\%\%\%\%\%) for $120 \mathrm{~ms}$, followed by a blank screen. Participants were instructed to respond as quickly and accurately as possible. Participants' vocal responses caused an asterisk to be displayed on the screen, and the experimenter pressed a key to indicate whether a response was incorrect or correct, and for correct responses, whether there was a premature or late triggering of the microphone. A $2000 \mathrm{~ms}$ inter-trial interval intervened before the next trial. Rest breaks were given every 50 trials.

\section{Results}

Filler trials were not included in the analyses. The primary analyses were those on the accuracy dependent variable, with analyses by participants reported. The effects in accuracy were additionally assessed in item analyses, with $F$ designated $F i$, and it is noted when effects significant by participants were not also significant by items. We also analyzed the naming latencies for correct responses. The latency data were preprocessed for the removal of errors (53\% of trials), microphone failures (1.11\% of trials), and latencies more than 3 standard deviations away from a participant's correct mean latency calculated separately for high- and low-frequency targets $(1.73 \%$ of trials).

A Distractor Frequency (high vs. low) $\times$ Target Frequency (high vs. low) $\times$ Prime Type (identical vs. unrelated) ANOVA was conducted on the accuracy data. The means are shown in Fig. 1. The main effect of distractor frequency was significant, $F(1,23)=10.18, M S E=199, \eta_{\mathrm{P}}{ }^{2}=.31$, with accuracy higher with high-frequency than with low-frequency distractors. The main effect of prime type was also significant, with a 36-point cost of identity primes ( $55 \%$ reduction in the unrelated prime accuracy), $F(1,23)=90.06, M S E=152, \eta_{\mathrm{P}}^{2}=.78$. The target frequency main effect was not significant $(F<1)$. The twoway Target Frequency $\times$ Prime Type interaction was not significant $(F<1)$, but the two-way interactions involving distractor frequency were significant, $F(1,23)=8.72, M S E$ $=63, \eta_{\mathrm{P}}{ }^{2}=.28$, for the Distractor Frequency $\times$ Target Frequency interaction, and $F(1,23)=8.70, M S E=108, \eta_{\mathrm{P}}{ }^{2}$ $=.27$, for the Distractor Frequency $\times$ Prime Type interaction. These interactions were qualified by a significant three-way interaction, $F(1,23)=4.57, M S E=71, \eta_{\mathrm{P}}^{2}=.17$. The threeway interaction was not significant by items $(F i<1)$, presumably reflecting the fact that target frequency is a between-items variable. As is evident in Fig. 1, the effect of distractor frequency was mainly evident for the unrelated prime condition. The simple interaction effects of Distractor Frequency $x$ Target Frequency were examined within each level of prime type. For the identity condition, accuracy was low in all conditions, and there was no significant interaction $(F<1)$. In fact, there was also no significant simple effect of target or distractor frequency in the identity prime condition. For the unrelated condition, there was a significant Distractor Frequency $\times$ Target Frequency interaction, $F(1,23)=13.01$, $M S E=66, \eta_{\mathrm{P}}{ }^{2}=.36$, with the benefit of a high-frequency distractor larger for high- than for low-frequency targets. Simple effects of distractor frequency within each level of target frequency revealed a substantial benefit for high distractor frequency with high-frequency unrelated targets, $F(1,23)=13.05, M S E=220, \eta_{\mathrm{P}}^{2}=.36$, whereas the benefit

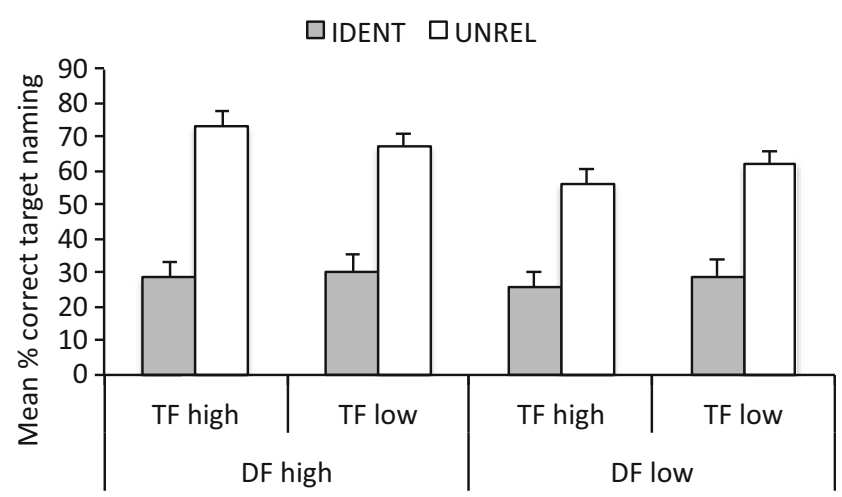

Fig. 1 Experiment 1: Mean percentage correct in the naming task as a function of distractor frequency, target frequency, and prime type (identity vs. unrelated). Error bars are standard errors of the mean 
Table 1 Experiment 1: Mean percentage of error responses that consisted of naming the distractor word, as a function of distractor frequency, target frequency ( $\mathrm{HF}=$ high frequency, $\mathrm{LF}=$ low frequency) and prime type $(N=9)$

Distractor frequency

\begin{tabular}{lllll}
\cline { 2 - 5 } & High & \multicolumn{3}{c}{ Low } \\
\hline Prime type & HF target & LF target & HF target & LF target \\
Identity & 100 & 96 & 98 & 94 \\
Unrelated & 90 & 72 & 87 & 79 \\
\hline
\end{tabular}

of a high-frequency distractor was not significant for lowfrequency unrelated targets, $F(123)=2.06, M S E=143, p=$ .17 .

Given the surprisingly large accuracy cost for the identity prime condition, a record of error responses was made for the last 13 participants, only nine of whom had errors in all cells. Table 1 shows for these nine participants the percentage of incorrect responses that consisted of naming the preceding distractor word instead of the target. A Distractor Frequency $\times$ Target Frequency $\times$ Prime Type ANOVA confirmed a main effect of prime type, with distractor responses constituting a higher proportion of errors on identity trials, $F(1,8)=10.42$, $M S E=386, \eta_{\mathrm{P}}{ }^{2}=.57$, and a main effect of target frequency, with more distractor responses as a proportion of errors on high- than on low-frequency words, $F(1,8)=10.84, M S E=$ $122, \eta_{\mathrm{P}}^{2}=.57$. There were no other effects.

The latency data had empty cells because of the high error rate, and analyses were conducted only by participants. Analysis for the participants with complete data sets revealed no effect of target frequency $(F<1)$. Consequently the data were pooled over target frequency, and the data for all participants were subjected to a Distractor Frequency $\times$ Prime Type ANOVA. The means are shown in Table 2. There were main effects of distractor frequency, $F(1,23)=7.79, M S E=19713$, $\eta_{\mathrm{P}}{ }^{2}=.25$, with faster responses when distractor frequency was high. There was also a main effect of prime type, $F(1,23)=$ $27.06, M S E=18715, \eta_{\mathrm{P}}^{2}=.54$, with longer latencies in the identity than the unrelated condition. There was no interaction $(F<1)$

Table 2 Experiment 1: Mean naming latencies (correct responses) and as a function distractor frequency and prime type

\section{Distractor frequency}

\begin{tabular}{lll}
\hline Prime type & High & Low \\
Identity & 1003 & 1101 \\
Unrelated & 876 & 938 \\
\hline
\end{tabular}

\section{Discussion}

Accuracy The results supported expectations that the intervening word priming paradigm would produce RB. The large magnitude of the effect was surprising. Unlike typical RSVP arrangements, the present priming task potentially conferred a disadvantage on the target by having the target duration less than that of the prime and distractor. Luo and Caramazza (1995) found an increase in RB when the duration of the first presentation of a repeated item $(\mathrm{C} 1)$ was increased. The effect was most marked when the duration was increased from $25 \mathrm{~ms}$ and $50 \mathrm{~ms}$ up to $100 \mathrm{~ms}$, and the authors attributed the effect on RB to increased effectiveness of the encoding of $\mathrm{C} 1$. The present results may mean that relatively better encoding of the prime than the target increased participants' uncertainty about whether the target was the same as the prime on identity trials.

With respect for evidence of interitem competition, distractor frequency had a robust main effect in the direction predicted by Morris et al. (2009). That is, target identification was poorer when the distractor was low frequency than when it was high frequency, consistent with the idea that the noisy activation footprint of less-well-learned words increases their ability to compete with other items for access to working memory. The effect was most evident for the unrelated prime condition. There was some evidence of floor compression in the identity condition, with nine of the 24 participants having an average accuracy of less than $15 \%$ on identity prime trials. The low accuracy in the identity condition indicates that participants were unlikely to offer the prime as a guess when they failed to identify the target.

Also, as predicted from the competition account (Morris et al., 2009), there was an interaction of distractor and target frequency, with accuracy a little higher for high-frequency targets when distractor frequency was high, and a reverse frequency effect (higher accuracy for low-frequency targets) when distractor frequency was low. This result indicates that more effective processing of high-frequency words may not be seen in the presence of interitem competition. On the Morris et al. (2009) model, a low-frequency adjacent word is a stronger competitor, and by extension, a high-frequency target is more vulnerable to competition.

With respect to the effect of frequency-based interitem competition on the RB, the predictions were not supported. Although there was a significant Distractor Frequency $x$ Prime Type interaction, the pattern was not as predicted. At low distractor frequency, RB was expected to be larger, but, in fact, RB was larger for a high-frequency distractor (40 percentage points) than for a low-frequency distractor (21 percentage points). Also, the three-way interaction of distractor frequency, target frequency and prime type was significant by participants, but it did not show the predicted pattern. This interaction arose because the effects of distractor and target 
frequency predicted from the competition model were mainly evident in the unrelated prime condition. Furthermore, RB was not larger for high-frequency targets with a lowfrequency distractor; RB was maximal for high-frequency targets with high-frequency distractors, reflecting the fact that unrelated-prime accuracy was highest in this condition. On the face of it, this result indicates that any effects of word frequency on word competitiveness do not play a role in the RB. However, it is difficult to draw this conclusion confidently because of possible floor compression in accuracy in the identity condition. Experiment 2 provided a better test of the role of distractor frequency in the RB because target accuracy improved when targets were displayed in upper case.

The analysis of error responses revealed that participants were highly likely to give the distractor as a response when their responses were incorrect. Participants did not necessarily believe that the distractor was a correct response; they may have preferred to guess an adjacent word rather than say "don't know." The distractor response was less frequent on unrelated trials, perhaps because the unrelated prime provided a choice of two responses other than the target. The finding that participants almost always gave the distractor as their response on identity trials reflects a strong RB effect and an associated bias against giving the prime as a guess. As suggested by Kanwisher (1987), when RB occurs, participants attribute the target's perceptual evidence to the prime.

The finding that distractor intrusion errors were more likely for high-frequency targets was unexpected. One possible explanation is that this result is driven mainly by the frequency of the prime (which was matched to target frequency). A highfrequency prime may produce a higher experienced familiarity or perceptual fluency (Whittlesea, Jacoby, \& Girard, 1990). As a result, participants may be more confident that they saw the prime first in item sequence and not in the target position.

Response latencies The accuracy results were supported by the latency data, in that low distractor frequency and identity primes slowed latencies. Notably, the RB effect was large (145 ms), and the latencies were long overall by comparison with typical lexical tasks such as naming and the LDT. These results confirm that target identification was difficult, and that participants experienced considerable uncertainty on identity trials.

\section{Experiment 2}

In Experiment 2, a second sample of students performed the Experiment 1 task with the same item set. The only difference was that the target was displayed in uppercase letters, with the prime and distractor remaining in lowercase letters. It was expected that RB would be reduced because the prime and target could be more easily distinguished. Additionally, overall accuracy might increase because of a reduction in perceptual masking by the preceding distractor.

\section{Method}

Participants Twenty-four undergraduate psychology students (20 females, mean age 19.12 years) participated for course credit. As noted, Experiments 1 and 2 were run concurrently.

Materials and design The lists were the same as in Experiment 1, except that target words were displayed in uppercase letters. The prime and distractor words remained in lower case.

Procedure The experiment was conducted in the same manner as Experiment 1. The proportion of errors that were distractor responses was collected for the last 14 participants.

\section{Results}

The accuracy data are shown in Fig. 2. A Distractor Frequency (high vs. low) $\times$ Target Frequency (high vs. low) $\times$ Prime Type (identity vs. unrelated) ANOVA revealed a main effect of distractor frequency, with accuracy again higher when the distractor was high frequency, $F(1,23)=21.31, M S E=124$, $\eta_{\mathrm{P}}^{2}=.48$. The main effect of prime type was also significant, reflecting a 12-point cost for identity primes (20\% of the unrelated condition accuracy), $F(1,23)=12.12, M S E=606, \eta_{\mathrm{P}}^{2}$ $=.35$. The only other significant effect was a Distractor Frequency $\times$ Target Frequency interaction, reflecting a larger effect of distractor frequency on high- than on low-frequency targets. Simple effects analysis of the distractor frequency effect within each level of target frequency indicated that the distractor frequency effect was significant for high-frequency targets, $F(1,23)=26.98, M S E=133, \eta_{\mathrm{P}}^{2}=.54$, but not for low-frequency targets, $F(1,23)=1.81, p=.19$. There was a trend for a Distractor Frequency $\times$ Prime Type interaction, $F(1,23)=2.87, M S E=177, p=.10 ; F i(1,126)=2.84$,

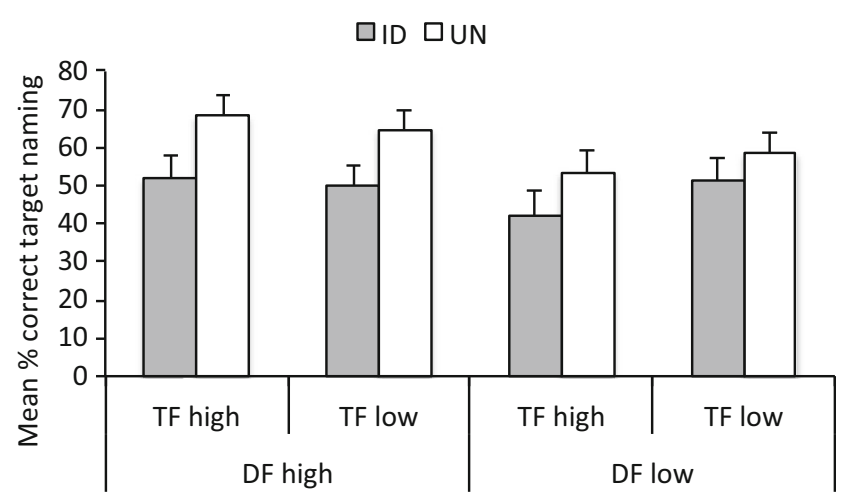

Fig. 2 Experiment 2: Mean percentage correct in the naming task as a function of distractor frequency, target frequency, and prime type (identity vs. unrelated). Error bars are standard errors of the mean 
Table 3 Experiment 2: Mean percentage of error responses that consisted of naming the distractor word, as a function of distractor frequency, target frequency ( $\mathrm{HF}=$ high frequency, $\mathrm{LF}=$ low frequency) and prime type $(N=12)$

Distractor frequency

\begin{tabular}{lllll}
\cline { 2 - 5 } & High & \multicolumn{3}{c}{ Low } \\
\hline Prime type & HF target & LF target & HF target & LF target \\
Identity & 98 & 97 & 95 & 95 \\
Unrelated & 84 & 71 & 81 & 76 \\
\hline
\end{tabular}

$M S E=413, p=.095$. As is evident in Fig. 2, this trend is mainly because the increase in accuracy when the distractor frequency was high was especially evident in the unrelated prime condition, in accord with the results of Experiment 1.

The percentage of errors that were distractor responses is shown in Table 3 for the 12 participants (of the 14 sampled) who had errors in all cells. In a Distractor Frequency $\times$ Target Frequency $\times$ Prime Type ANOVA, only the main effect of prime type was significant, with a higher proportion of distractor responses given in error on identity prime trials, $F(1,11)=34.06, M S E=238, \eta_{\mathrm{P}}^{2}=.76$.

The latency data were preprocessed as before, with a loss of $45 \%$ of trials as errors, $0.72 \%$ as microphone failures, and $2.02 \%$ as extreme latencies. There was a nonsignificant target frequency effect (shorter latencies for high-frequency targets, $p=.1)$ in the data for the 23 participants with complete data. As in Experiment 1, the latencies were pooled over target frequency, and all participants' mean latencies were subjected to a Distractor Frequency $\times$ Prime Type ANOVA. There were significant main effects of these variables (see Table 4). As in Experiment 1, responses were faster when distractor frequency was high, $F(1,23)=14.63, M S E=7920, \eta_{\mathrm{P}}{ }^{2}=.39$, and there was an identity priming cost, with slower responses on identity prime trials, $F(1,23)=8.70, M S E=17,457, \eta_{\mathrm{P}}{ }^{2}=.27$. The latency cost was nonsignificantly larger when distractor frequency was low ( $p=.09$ for the interaction).

Cross-experiment analysis In order to compare the findings over the two experiments, an Experiment (1 vs. 2) $\times$ Distractor Frequency $\times$ Target Frequency $\times$ Prime Type ANOVA was conducted on the accuracy data. As before, there were main effects of distractor frequency and prime type, $\eta_{\mathrm{P}}{ }^{2}=.39$ and

Table 4 Experiment 2: Mean naming latencies (for correct responses) as a function distractor frequency and prime type

\section{Distractor frequency}

\begin{tabular}{lll}
\hline Prime type & High & Low \\
Identity & 838 & 928 \\
Unrelated & 779 & 828 \\
\hline
\end{tabular}

$\eta_{\mathrm{P}}{ }^{2}=.65$, respectively, as well as a Distractor Frequency $\times$ Target Frequency interaction, $\eta_{\mathrm{P}}^{2}=.30$. The Distractor Frequency $\times$ Prime Type interaction, which fell short of significance in Experiment 2, was significant in the combined analysis, $\eta_{\mathrm{P}}^{2}=.18\left(\eta_{\mathrm{P}}^{2}=.22\right.$ in the items analysis $)$. The three-way interaction of distractor frequency, target frequency, and prime type, which was significant only in Experiment 1, was significant by participants in the combined analysis, $\eta_{\mathrm{P}}^{2}=$ .02 , but not by items. The three-way interaction was decomposed as simple Distractor Frequency $\times$ Target Frequency interaction tests for each prime condition. The frequency interaction was significant both for the identity condition $F(1,46)=4.44, M S E=106, \eta_{\mathrm{P}}^{2}=.09$, and for the unrelated condition, $F(1,46)=15.88, M S E=78, \eta_{\mathrm{P}}^{2}=.26$, with both effects also significant by items. The four-way interaction was not significant. The only significant interaction involving experiment was that the repetition cost was larger in Experiment 1 than Experiment 2, $F(1,46)=20.75, M S E=650, \eta_{\mathrm{P}}{ }^{2}=.31$ for the Prime Type $\times$ Experiment Interaction ( $p=.09$ by items). The main effect of experiment was not significant.

\section{Discussion}

The results of Experiment 2 were in many respects similar to those of Experiment 1. Target accuracy in Experiment 2 again showed an advantage for high-frequency distractors and unrelated primes. As in Experiment 1, the distractor frequency effect was mainly evident for high-frequency targets, although the interaction fell short of statistical significance. The notable change in the results of Experiment 2 was that accuracy was substantially higher in the identity prime condition, and RB was smaller than in Experiment 1. The latency data were in line with Experiment 1 in that there was a cost of repetition and high distractor frequency. The latencies were shorter in Experiment 2 than Experiment 1, as confirmed by a post hoc test, $F(1,46)=6.43, M S E=138,708, \eta_{\mathrm{P}}{ }^{2}=.12$, consistent with the reduction in difficulty from the target letter-case change.

In Experiment 1, as noted, accuracy on identity prime trials may have been affected by floor compression. As a result of the improvement in performance in the identity condition in the present experiment, the effects of distractor and target frequency were somewhat evident in this condition. Notably, there was a similarity in the pattern of means for the identity condition in the two experiments. Specifically, in both experiments, accuracy was lower, compared with the other three conditions, for high-frequency targets with a low-frequency distractor. The Distractor Frequency $\times$ Target Frequency interaction was significant in the identity condition in the crossexperiment analysis. The same pattern was more strongly evident in the unrelated prime condition in both experiments. This result is consistent with frequency-driven competition effects on target accuracy, according to which high- 
frequency targets are more vulnerable to competition and lowfrequency distractors compete more.

In terms of competitor effects, other things being equal, a low-frequency target with a high-frequency distractor should have the highest accuracy. However, the typically observed identification advantage for better learned high-frequency words (Monsell, 1991) must also be taken into account. In line with predictions, the standard frequency effect favoring high-frequency words was somewhat more evident when distractors were high frequency, and thus less competitive.

Concerning the role of competition in $\mathrm{RB}$, the magnitude of RB was not affected by distractor frequency in Experiment 2 . There was a trend for a Distractor Frequency $\times$ Prime Type interaction, and it was significant in the cross-experiment analysis. However, as in Experiment 1, the trend was not in the predicted direction. Contrary to expectation, RB was numerically larger with high-frequency distractors. In the latency data, there was a trend for the RB cost to be larger with lowfrequency distractors, as predicted. However, the interaction was not significant $(p=.09)$, and in any case, the latency data may not provide a direct measure of RB.

The effect of a letter-case change is surprising in the context of early research that found substantial RB when visual similarity is not preserved across item repeats (Bavelier, 1994), with Kanwisher (1987) finding that RB was preserved over letter case changes. RB was observed in Experiment 2 with a target case change, but its magnitude was markedly reduced. The effect of letter-case changes may be more marked in the present paradigm in which only three words were presented on each trial. In addition, recent evidence (Brill, Glass, Rashid, \& Hussey, 2008) suggests that the effect of a case change may have been underestimated in Kanwisher's (1987) experiment.

The case-change effect in Experiment 2 can be interpreted in terms of distinctiveness during either perceptual or memory processes. At the perceptual level, there might be a reduction in the ability of the lowercase distractor to mask the uppercase target because some uppercase letters would extend beyond the envelope of the lowercase word. There should be no change in the masking between the prime and the distractor, both of which remained in lower case. The clearest test of the possibility of reduced masking by the distractor on an uppercase target is to compare accuracy on unrelated trials, given that this comparison is not impacted by the RB effects of different magnitudes in the two experiments. In fact, accuracy on unrelated target trials was no higher in Experiment $2(61 \%)$ than in Experiment 1 (65\%). Because participants were randomly assigned to the experiments, this comparison is not contaminated by sampling differences. It is clear that the improvement in accuracy in Experiment 2 was entirely on identity prime trials. Perceptual masking is not relevant to the identity condition because the prime and target are not temporally adjacent. Additionally, it is unlikely that positive priming of the target's type representation in the identity condition played a role in the change in results over the experiments. A case change between prime and target can be expected to leave unchanged, or reduce, any priming benefits arising in lexical processing.

Another possibility is that the competitive effects of the distractor are reduced when the target is displayed in upper case. This possibility is unlikely, given that competition is thought to be located at a stage after letter processing, namely, in the activation of word's memory representation. Furthermore, a reduction in competition can be expected to increase accuracy on unrelated trials in Experiment 2. As noted above, there was no change in unrelated trial accuracy between the experiments.

In sum, it seems more likely that that the large increase in accuracy when primes and targets are presented in different letter case arises later in the processing sequence, and uniquely for the identity priming condition. Most plausibly, the perceptual difference between the prime and target enhances token distinctiveness. This would allow participants to more easily establish the prime and target as separate events.

\section{General discussion}

Summary The results of the two experiments together established that target accuracy is lower when the prior distractor is low frequency than when the distractor is high frequency, consistent with stronger competition by lowfrequency adjacent words for access to awareness. Also consistent with the competition account (Morris et al., 2009), the large and robust advantage for high-frequency words that is typically reported in lexical processing tasks was reversed for unrelated targets in both experiments when the target was preceded by a low-frequency distractor. This is predictable from the proposal that low-frequency words compete better than high-frequency words, so that a high-frequency target should suffer more than a low-frequency target from the competitive effects of a low-frequency distractor. The implication is that the typical word-frequency effect can be obscured by other factors when targets are displayed briefly and embedded in a short word sequence.

When the prime was the same word as the target, a robust RB effect was seen, even though participants were only required to name the last word. Kanwisher's (1987) assumption that tokenization is not required for last-word report may be incorrect, so this result does not necessarily challenge a role for tokenization impairments in RB. When participants failed to identify the target, they often gave the distractor word as a response, especially on identity prime trials. There was no evidence that the magnitude of RB depends upon targetword frequency. The magnitude of RB was decreased in Experiment 2 by presenting the target in a different letter case 
from the prime and distractor, presumably because the prime and target events were distinguished by the case change. Finally, a prediction of the competition model account of RB was not supported: The magnitude of RB was not greater with a low-frequency than a high-frequency distractor.

Support for the competition model The present results provide support for the competition model in terms of adjacent-word frequency effects (Morris et al., 2009). The frequency-based effects of the intervening distractor were clearly evident for unrelated targets. However, we failed to find specific evidence for a role of interitem competition in the $\mathrm{RB}$, in that $\mathrm{RB}$ was not greatest for a low-frequency distractor with a high-frequency target. This result is a problem for the model, but perhaps not decisively. In Experiments 1 and 2 accuracy was lowest in this frequency cell in the identity condition (significantly so in Experiment 2). The magnitude of RB was not correspondingly large because the unrelated targets also suffered an accuracy cost. It is possible that the effects of frequency-based competition on the RB will depend upon overall accuracy.

In a similar vein, the authors noted that according to their model, the overall magnitude of RB determines whether RB will be moderated by the frequency of the targets $\mathrm{C} 1$ and $\mathrm{C} 2$. In their simulations with a pattern mask as an adjacent item, Morris et al. (2009) found that low-frequency repeated words are more likely than high-frequency repeated words to outcompete an adjacent item when RB is not large. When RB is large, the increment in competitiveness of a low-frequency word does have a sufficiently large impact for the word to out-compete the mask and be reported more often than a high-frequency word. In the present case, it is arguable that the loss of competitiveness of a repeated high-frequency word with a low-frequency distractor will only increase RB when the competitive cost for unrelated targets is modest, and repeated-item accuracy is not substantially compromised.

There is an alternative explanation of the effect of distractor frequency that must be addressed. Because low-frequency words are identified less easily and more slowly than highfrequency words are (Burt, Howard, \& Falconer, 2011; Monsell, 1985) it is arguable that there is a larger spillover cost onto the target from low-frequency than high-frequency distractors. Given the brief target duration, the spillover effect should be substantial, especially for a low-frequency target after a low-frequency distractor. The response latencies provided some support for spillover in that latencies were longer after low-frequency distractors. However, with respect to accuracy, there is a problem. Although spillover would explain the main effect of distractor frequency, it would not explain the reverse effect of target frequency that was observed for unrelated targets (in both experiments) with low-frequency distractors, in which low-frequency targets had higher accuracy than high-frequency targets had. Spillover predicts that the typical advantage for high-frequency targets should be increased by low- compared with high-frequency distractors, a result opposite to what was observed for unrelated targets.

Is RB due to competition? Frequency-based word competition effects have been demonstrated in the present experiments. Consequently, the present results suggest that interitem competition affects participants' ability to report targets in the RSVP task. We found no direct evidence for the theory of Morris et al. (2009) that $\mathrm{RB}$ results from reduced competitiveness from a repeated target, but a role of competition in $\mathrm{RB}$ remains viable. For example, a role of competition in $\mathrm{RB}$ is consistent with the larger RB observed by Whittlesea and Masson (2005) when an intervening item was a new word rather than the same intervening word on every trial or a symbol string.

The present and previous results do suggest one strong conclusion about the role of interitem competition in RB. Specifically, it is unlikely that competition is the sole basis of RB effects. In Experiment 2, a target letter-case change substantially ameliorated RB when there is no compelling reason why a case change should affect competition, and no evidence of any change in competition effects on unrelated trials. Additionally, RB-like effects have been demonstrated in word-priming paradigms with briefly displayed targets and no intervening word between the prime and the target (Burt et al., 2014; Hochhaus \& Marohn, 1991; Huber, 2008). When an identity prime competes with a target, then the correct response should access working memory regardless of which item wins the competition. It might be argued that these repetition costs in priming tasks are different from RB as observed in RSVP sequences, but parsimony dictates otherwise. Repetition costs appear to be general across a variety of reporting requirements, including report of the last word only, report of all target words, and decisions about repetitions (Kanwisher \& Potter, 1990; Luo \& Caramazza, 1995; Whittlesea \& Masson, 2005), although RB is not always observed in last-word report (Kanwisher, 1987; Masson, 2004). $\mathrm{RB}$ also appears to be general across a variety of paradigms, including RSVP sentences, short- and long-word sequences, target-plus-distractor and target-only sequences, and between orthographically similar words as well as repetitions (Bavelier et al., 1994; Bond \& Andrews, 2008; Harris \& Morris, 2001; Masson, 2004). The primary requirement for RB seems to be that the to-be-reported word(s) must be displayed briefly; RB reverses to facilitatory priming in lexical tasks when a target word remains on-screen until a participant responds (Burt et al., 2014). Overall, the available evidence is consistent with the view that RB is multiply determined, with competitive effects of adjacent stream items perhaps contributing to RB, but not its sole cause.

Tokenization and RB Although Kanwisher (1987) assumed that tokenization was not required if participants were asked to report only the last word in an RSVP stream, it is likely that 
this assumption is incorrect, at least in some cases. Experience at the present task and participant reports suggest that one source of difficulty is deciding which word came last in the sequence. In other words, events must be registered and ordered before a response is selected. The reduction in RB with a case change for the target in Experiment 2 indicates that separation of the prime and target events on identity prime trials is facilitated when the prime and target are perceptually distinct. Tokenization and event ordering are not necessarily completed online during the RSVP, as proposed by Kanwisher and Potter (1989). Resolving evidence for a target word into one or two occurrences may continue at the end of the stream. Research conducted within reconstructive accounts of RB has provided evidence that RB can be affected by events following a target in the RSVP stream or by the nature of the task instruction that is given at the end of the stream (Masson, Caldwell, \& Whittlesea, 2000; Whittlesea, Masson, \& Hughes, 2005). The large effect of prime identity on response latencies in the present experiments is consistent with a period of event reconstruction after target presentation.

Response biases and RB When participants were unable to report the target on identity trials they almost always gave the distractor item as a response. This behavior appeared to be a consequence of $\mathrm{RB}$ rather than due to participants' perceiving the distractor as the target. It appears that participants choose another word as a response when they have not successfully identified the target as the same word as the prime. In unpublished work we have found that when participants fail to report the target they are not highly likely to choose the distractor if they are given a choice between the distractor and a new unrelated word.

In previous work, in a priming paradigm without any intervening word, we suggested that uncertainty about repetitions caused participants to show a bias against deciding that the target was the same as the prime (Burt et al., 2014). This presumed bias was exacerbated by our use of a choice task (LDT), in which a bias could be expressed as a nonword response, and by our use a long prime duration, which may increase confidence about the identity of the prime and thereby increase uncertainty about the perceptual evidence from the brief target. In the present experiments, there was no choice response, and the prime duration, although longer than the target duration, was relatively short, at $120 \mathrm{~ms}$. In addition, the intervening distractor word in the present experiments distinguished the prime and target as separate events. Overall, biases against the prime appear to be a consequence of confusion between prime and target rather than a primary cause of $\mathrm{RB}$.

Refractoriness Recent findings have been interpreted as evidence that RB reflects a brief period of refractoriness in an identified target word's type representation (Bond \& Andrews, 2008; Huber, 2008; Luo \& Caramazza, 1996). RB increases when the duration of the first presentation of the repeated word is increased (Huber, 2008; Luo \& Caramazza, 1995), a result consistent with the idea that refractoriness increases with the duration or effectiveness of word encoding. The present results are inconsistent with the theory that RB is caused by refractoriness because a letter-case change for the target markedly reduced RB. Refractoriness is evoked during the processing of the prime word and should exert similar effects on uppercase and lowercase targets. Furthermore, if refractoriness affects the identification of a repeated word, it is difficult to explain the robust facilitatory effects of word repetition on response latencies in lexical tasks (Burt, Mardle, \& Humphreys, 1996; Forster \& Davis, 1984; Kirsner \& Speelman, 1996).

Priming versus RSVP paradigms The present task differed from priming tasks typical in lexical processing by having an intervening word between the prime and target and a brief duration for the target. The task differed from the traditional RSVP task in that the word sequence was shorter than is typical, and participants were required to report only the final word. The robust RB observed in both experiments accords with a common view that the RB arises from difficulty in registering and ordering events under time pressure. It also suggests why making the target available until a participant responds is important for priming facilitation. Specifically, the longer duration allows participants to avoid any ambiguity about which word is the target and to reduce or eliminate uncertainty about whether a relatively brief unmasked prime is the same word as the target. It is likely that facilitation would be observed in the present paradigm if the target duration were lengthened by several hundred ms. In a masked priming paradigm, identity priming facilitation has been observed with a long-duration target and a visible intervening word (Forster, 2009, 2013).

In conclusion, the present experiments are consistent with a major role of limits under time pressure on tokenization and event ordering in RB, even when participants report only the last word in a short word sequence. The results also provide compelling evidence of frequency-dependent competition between a target and an immediately preceding distractor word. The role of frequency-dependent competition in RB awaits further research.

Author note The experiments were conducted by Jessica Jolley as partial completion of her honors degree in psychology at the University of Queensland.

\section{References}

Bavelier, D. (1994). Repetition blindness between visually different items: The case of pictures and words. Cognition, 51(3), 199-236. doi:10.1016/0010-0277(94)90054-X 
Bavelier, D., Prasada, S., \& Segui, J. (1994). Repetition blindness between words: Nature of the orthographic and phonological representations involved. Journal of Experimental Psychology: Learning, Memory, and Cognition, 20(6), 1437-1455. doi:10.1037/02787393.20.6.1437

Bond, R., \& Andrews, S. (2008). Repetition blindness in sentence contexts: Not just an attribution? Memory \& Cognition, 36(2), 295-313. doi:10.3758/mc.36.2.295

Brill, G. A., Glass, A. L., Rashid, H., \& Hussey, E. (2008). Visual identity and uncertainty in repetition blindness. The American Journal of Psychology, 121(3), 409-449. doi:10.2307/20445475

Burt, J. S., Howard, S., \& Falconer, E. K. (2011). T1 difficulty affects the AB: Manipulating T1 word frequency and T1 orthographic neighbor frequency. Attention, Perception, \& Psychophysics, 73(3), 751765. doi:10.3758/s13414-010-0054-x

Burt, J. S., Kipps, T. J., \& Matthews, J. R. (2014). Repetition in visual word identification: Benefits and costs. Quarterly Journal of Experimental Psychology, 67(10), 1986-2009. doi:10.1080/ 17470218.2014 .896386

Burt, J. S., Mardle, L., \& Humphreys, M. S. (1996). Expectancy-based associative and identity priming in pronunciation. Australian Journal of Psychology, 48, 64-74. doi:10.1080/ 00049539608259508

Chialant, D., \& Caramazza, A. (1997). Identity and similarity factors in repetition blindness: Implications for lexical processing. Cognition, 63(1), 79-119. doi:10.1016/S0010-0277(96)00789-5

Forster, K. I. (2009). The intervenor effect in masked priming: How does masked priming survive across an intervening word? Journal of Memory and Language, 60(1), 36-49. doi:10.1016/j.jml.2008.08. 006

Forster, K. I. (2013). How many words can we read at once? More intervenor effects in masked priming. Journal of Memory and Language, 69(4), 563-573. doi:10.1016/j.jml.2013.07.004

Forster, K. I., \& Davis, C. (1984). Repetition priming and frequency attenuation in lexical access. Journal of Experimental Psychology: Learning, Memory, and Cognition, 10(4), 680-698. doi:10.1037/ 0278-7393.10.4.680

Grill-Spector, K., Henson, R., \& Martin, A. (2006). Repetition and the brain: Neural models of stimulus-specific effects. Trends in Cognitive Sciences, 10(1), 14-23. doi:10.1016/j.tics.2005.11.006

Harris, C. L., \& Morris, A. L. (2001). Identity and similarity in repetition blindness: No cross-over interaction. Cognition, 81(1), 1-40. doi:10. 1016/s0010-0277(00)00138-4

Hochhaus, L., \& Marohn, K. M. (1991). Repetition blindness depends on perceptual capture and token individuation failure. Journal of Experimental Psychology: Human Perception and Performance, 17(2), 422-432. doi:10.1037/0096-1523.17.2.422

Huber, D. E. (2008). Immediate priming and cognitive aftereffects. Journal of Experimental Psychology: General, 137(2), 324-347. doi:10.1037/0096-3445.137.2.324

Kanwisher, N. G. (1987). Repetition blindness: Type recognition without token individuation. Cognition, 27(2), 117-143. doi:10.1016/00100277(87)90016-3

Kanwisher, N. G., \& Potter, M. C. (1989). Repetition blindness: The effect of stimulus modality and spatial displacement. Memory \& Cognition, 17(2), 117-124.

Kanwisher, N. G., \& Potter, M. C. (1990). Repetition blindness: Levels of processing. Journal of Experimental Psychology: Human
Perception and Performance, 16(1), 30-47. doi:10.1037/00961523.16.1.30

Kilgarriff, A. (1995, November 3). BNC database and word frequency lists. Retrieved from http://www.kilgarriff.co.uk/bnc-readme.html

Kirsner, K., \& Speelman, C. (1996). Skill acquisition and repetition priming: One principle, many processes? Journal of Experimental Psychology: Learning, Memory, and Cognition, 22, 563-575. doi: 10.1037/0278-7393.22.3.563

Luo, C. R., \& Caramazza, A. (1995). Repetition blindness under minimum memory load: Effects of spatial and temporal proximity and the encoding effectiveness of the first item. Perception \& Psychophysics, 57(7), 1053-1064.

Luo, C. R., \& Caramazza, A. (1996). Temporal and spatial repetition blindness: Effects of presentation mode and repetition lag on the perception of repeated items. Journal of Experimental Psychology: Human Perception and Performance, 22(1), 95-113. doi:10.1037/ 0096-1523.22.1.95

Masson, M. E. J. (2004). When words collide: Facilitation and interference in the report of repeated words from rapidly presented lists. Journal of Experimental Psychology: Learning, Memory, and Cognition, 30(6), 1279-1289. doi:10.1037/0278-7393.30.6.1279

Masson, M. E. J., Caldwell, J. I., \& Whittlesea, B. W. A. (2000). When lust is lost: Orthographic similarity effects in the encoding and reconstruction of rapidly presented word lists. Journal of Experimental Psychology: Learning, Memory, and Cognition;Journal of Experimental Psychology: Learning, Memory, and Cognition, 26(4), 1005-1022. doi:10.1037/02787393.26.4.1005

Monsell, S. (1985). Repetition and the lexicon. In A. W. Ellis (Ed.), Progress in the psychology of language (Vol. 2, pp. 147-195). Hillsdale: Erlbaum.

Monsell, S. (1991). The nature and locus of word frequency effects in reading. In D. Besner \& G. W. Humphreys (Eds.), Basic processes in reading: Visual word recognition (pp. 148-197). Hillsdale: Erlbaum.

Morris, A. L., Still, M. L., \& Caldwell-Harris, C. L. (2009). Repetition blindness: An emergent property of inter-item competition. Cognitive Psychology, 58(3), 338-375. doi:10.1016/j.cogpsych. 2008.08.001

Schneider, W., Eschman, A., \& Zuccolutto, A. (2002). E-Prime user's guide. Pittsburgh: Psychology Software Tools.

Whittlesea, B. W. A., Dorken, M. D., \& Podrouzek, K. W. (1995). Repeated events in rapid lists: I. Encoding and representation. Journal of Experimental Psychology: Learning, Memory, and Cognition, 21(6), 1670-1688. doi:10.1037/0278-7393.21.6.1670

Whittlesea, B. W. A., Jacoby, L. L., \& Girard, K. (1990). Illusions of immediate memory: Evidence of an attributional basis for feelings of familiarity and perceptual quality. Journal of Memory and Language, 29(6), 716-732. doi:10.1016/0749-596X(90)90045-2

Whittlesea, B. W. A., \& Masson, M. E. J. (2005). Repetition Blindness in rapid lists: Activation and inhibition versus construction and attribution. Journal of Experimental Psychology: Learning, Memory, and Cognition, 31(1), 54-67. doi:10.1037/0278-7393.31.1.54

Whittlesea, B. W. A., Masson, M. E. J., \& Hughes, A. D. (2005). False memory following rapidly presented lists: The element of surprise. Psychological Research/Psychologische Forschung;Psychological Research/Psychologische Forschung, 69(5/6), 420-430. doi:10. 1007/s00426-005-0213-1 